\title{
Projetos de Educação para a Saúde em meio escolar: Da avaliação às práticas de referência
}

\author{
Maria Leonor da Costa Dias
}

Agrupamento de Escolas Viseu Norte, Viseu, Portugal

Maria João de Miranda Nazaré Loureiro

Universidade de Aveiro, Portugal

Maria Isabel Guedes Loureiro

Universidade Nova de Lisboa, Portugal

\begin{abstract}
Resumo
Planear intervenções pertinentes e eficazes no âmbito da Educação para a Saúde (EpS) em meio escolar determina a implementação de procedimentos de avaliação que traduzam, não só um balanço da ação desenvolvida, mas contribuam, igualmente, para a reflexão em torno do processo de implementação da EpS na realidade escolar portuguesa, com vista à otimização qualitativa das intervenções. À luz deste pressuposto, e visando estabelecer uma ligação entre a análise teórica e a reflexão a partir de uma prática concreta, o presente artigo tem como objetivos: (i) apresentar o modelo avaliativo instituído no estudo multicasos de índole qualitativa "Escola, Saúde e Sociedade: Estudos de Avaliação de Projetos de Educação para a Saúde", que se desenvolveu entre 2009/2011; (ii) divulgar as práticas de referência usadas num dos Projetos de Educação para a Saúde envolvidos no estudo, o qual evidenciou os critérios de qualidade que podem levar a considerá-lo como um 'bom' projeto.
\end{abstract}

Palavras-chave

Escola; Projetos de Educação para a Saúde; Avaliação; Práticas de referência

\section{Introdução}

Analisando os últimos cinco anos de implementação dos projetos de Educação para a Saúde (PEpS) nas escolas portuguesas, constata-se que os 
estudos de avaliação efetuados são reduzidos e fornecem, sobretudo, dados quantitativos. Entende-se, tal como Aldinger et al. (2008), Lee, Leger, \& Moon (2005), Mandim (2007) e Warwick et al. (2005), entre outros, que é necessário analisar as dinâmicas de implementação destes projetos, tendo em vista perceber as práticas de referência que desencadeiam a qualidade da sua ação e fornecer recomendações que estimulem a sua adoção.

A ideia de projeto tem subjacente uma dimensão prospetiva de antecipação, de representação de um real possível (Barbier, 1996), num processo de construção de algo que se vislumbra como possível e desejável. A dinâmica de participação, de negociação, de construção e de desenvolvimento de um projeto deverá traduzir-se numa reflexão contínua sobre os objetivos definidos, sobre o que é necessário redefinir, o que importa manter e o que pode ser importante alterar. Para tal, é necessário instituir procedimentos avaliativos, que se consagram com carácter formativo, que ajudem a otimizar a qualidade da sua ação.

Apesar de se admitir a importância da avaliação, quer interna, quer externa dos projetos, é forçoso reconhecer que apenas um número limitado a comporta, verificando-se o predomínio de processos quantitativos em detrimento da experimentação de novas abordagens e técnicas (Capucha, 2008; Costa, 2007; Nóvoa, Almeida, \& Le Boterf, 1993). Na verdade, existe o risco de procurar reduzir a avaliação de projetos a um método mais preocupado em medir a coerência interna do que em confrontar os seus resultados com a realidade a transformar, isto é, proceder à análise qualitativa dos "efeitos reais ou potenciais de um projeto" (Capucha, 2008; Hernando, 2009; Nóvoa et al., 1993).

A avaliação externa dos PEpS, que se preconizou no estudo "Escola, Saúde e Sociedade: Estudos de Avaliação de Projetos de Educação para a Saúde", foi alicerçada sobre uma démarche de referencialização, numa perspetiva de avaliação qualitativa, holística (Barbier, 1996; Capucha, 2008; Cardinet, 1976) e interativa, do ponto de vista dos atores e do investigador. 0 estudo analisou as dinâmicas de implementação e o desenvolvimento de cinco PEpS, criados nos Agrupamentos de Escolas desde 2005, na procura de práticas de referência, tendo por base as caracterizadas no Referencial de Avaliação construído. Assentou em questões previamente formuladas: Como avaliar práticas de referência no que respeita às dinâmicas de implementação 
de PEpS? Em que medida as dinâmicas de implementação de PEpS se constituem como práticas de referência? Que elementos emergem da avaliação de práticas de implementação de PEpS, consideradas de referência, que podem constituir-se como recomendações gerais para a sua divulgação e adoção?

Considerando a definição de boas práticas (best practices) em EpS preconizada por Kahan \& Goodstadt (1998) como sendo as práticas que desencadeiam intervenções eficazes na promoção da saúde, utilizou-se, em detrimento de boas práticas, a terminologia práticas de referência, considerando-as como práticas 'ideais', constituindo-se como referentes para intervenções na promoção da saúde. Apresentam características próprias em função da sua efetividade/eficácia para um determinado contexto, podendo ser aplicadas a outros contextos, devidamente adaptadas.

Não sendo possível divulgar num só artigo todo o trabalho realizado, optou-se, no presente artigo, por analisar, na contextualização teórica, o conceito de avaliação de projeto e os modelos avaliativos passíveis de implementação em PEpS. Explicita-se, adiante, a metodologia instituída no estudo. Aborda-se, na démarche de referencialização, a conceptualização e operacionalização da avaliação do objeto em estudo - PEpS, o qual foi analisado à luz do conceito de Escola Promotora de Saúde (EPS), expondo, no essencial, o Referencial de Avaliação construído. A finalizar, apresentamse, nos resultados, as práticas de referência das dinâmicas de implementação do PEpS do Agrupamento de Escolas A, o qual evidenciou, entre os cinco analisados, critérios de qualidade, sendo-Ihe reconhecida a classificação de um 'bom' projeto.

\section{Contextualização teórica}

\subsection{Avaliação de projetos}

A metodologia de projeto tem vindo a assumir um papel cada vez mais determinante na conceção, planificação e realização de atividades nas organizações educacionais. Entendidas como organizações dotadas de significativa margem de autonomia, instituem projetos das mais variadas índoles no sentido de promover a inovação e a mudança. 
Mendonça (2002) explicita a noção de projeto como:

(...) um processo consciente concretiza-se pela identificação de uma tensão diferencial, entre o que se deseja e o que se faz. Possui por isso uma finalidade. Prevê um certo número de meios para atingir essa finalidade. Precisa-se sob a forma de um plano de atividades sucessivas e significativas. Integra um produto que é avaliado (p. 17).

Por conseguinte, o projeto é um conjunto de operações eminentemente úteis e práticas que visam uma intervenção direcionada num determinado espaço de tempo, que deve obedecer a quatro princípios gerais: unidade na elaboração e na realização; singularidade da situação a melhorar; exploração de oportunidades em ambiente aberto; gestão da complexidade e incerteza da situação. Mendonça (2002) chama, ainda, a atenção para três aspetos que considera significativos: o seu caráter "exemplar", que se distancia do banal e do rotineiro para se preocupar com o idealizado e inédito; o seu caráter "operacional", concretizando-se de uma ou de outra forma; o seu caráter "personificador", que está ligado a alguém (um indivíduo, um grupo) que o determina, o orienta e o organiza.

Do acima referido, emerge um conjunto de características inerentes ao(s) projeto(s), das quais se salienta: "Intencionalidade" - significando que o projeto implica intenção e depende do envolvimento e do empenho dos intervenientes numa visão partilhada; "Responsabilidade e autonomia dos intervenientes" - na medida em que estes são construtores do seu desenvolvimento/ação; "Autenticidade" - no sentido em que o projeto deve equacionar um problema relevante e pertinente para a comunidade e, ao mesmo tempo, para os seus intervenientes; "Complexidade" - integra múltiplas dimensões, sejam elas internas e/ou externas, individuais e/ou coletivas, socioculturais; "Criatividade" - dado que é necessário encontrar ideias novas; "Um processo e um produto" - o projeto é uma construção progressiva, que tem um início, um meio e um fim que se interligam (fases do projeto). Ao ser avaliado, pode dar origem a novas dúvidas ou problemas que justifiquem a (re)formulação da sua ação.

Hadji (1994) define a avaliação como:

(...) o acto pelo qual se formule um juízo de "valor" incidindo num objecto determinado (indivíduo, situação, acção, projecto, etc.) por meio de um confronto entre duas séries de dados que são postos em relação: dados que são de ordem do facto em si e dizem respeito ao objecto real a avaliar; dados 
que são de ordem do ideal e que dizem respeito a expectativas, intenções ou projectos que se aplicam ao mesmo objecto (p. 31).

Nesta linha, também Rodrigues (1995) descreve a avaliação como o confronto entre "dados de facto", o real, o construído que constitui o referido, com o desejado, o ideal - o referente, composto de normas, objetivos ou critérios, e permite atribuir um valor, uma utilidade ou uma significação aos dados concretos. Assim, avaliar um projeto é comparar o construído com um referencial de avaliação, o qual integra o ideal desejável para o projeto.

Consigna-se, no entanto, em concordância com Figari (1996), que a avaliação de um projeto é mais do que o estabelecimento de uma relação ou mesmo o calcular a distância entre o referente e o referido. Como defende Figari (1996), avaliar "é sobretudo reflectir sobre esse afastamento" (p. 44) para que se possa compreender os significados e os sentidos do projeto e contribuir para o incremento da sua qualidade.

\subsubsection{Avaliação processual de projetos de Educação para a Saúde}

Planear intervenções pertinentes e eficazes no âmbito da ação do PEpS com vista ao fortalecimento do contexto em que se insere deverá envolver sempre uma antevisão de resultados (Barbier, 1996). Para tal, é necessário definir os critérios de sucesso esperados, que são o reflexo dos desejos e expectativas dos vários intervenientes, garantindo referenciais que integrem o ideal desejável para o projeto (Figari, 1996). Estes permitirão a avaliação/monitorização da sua pertinência, coerência, eficiência e efetividade ao longo da sua construção/percurso e, no final do mesmo, a apreciação do impacto da sua ação na capacitação dos alunos. Ao obter-se um conjunto de informações torna-se possível a reflexão, de forma a compreender os significados e os sentidos do projeto e emitir um juízo de valor.

Para que os PEpS cumpram o requisito de terem em conta as situações reais e serem relevantes para dar resposta aos problemas existentes, é necessário realizar um diagnóstico que sirva de base às opções que se fazem. A avaliação diagnóstica constitui, assim, um ponto de partida para a conceção e desenvolvimento de qualquer projeto desta natureza. 
Casado (2000) e Hernando (2009) consideram que, em função da finalidade da avaliação de PEpS, esta pode ser formativa ou sumativa. A avaliação formativa é realizada durante a ação do projeto e tem como principal objetivo a melhoria e o aperfeiçoamento deste. A avaliação sumativa efetua-se no final e tem por objetivo tanto a melhoria como a sua contabilidade, e ainda a análise do impacto da sua ação junto dos seus destinatários.

A avaliação qualitativa de PEpS é um processo que aplica estratégias rigorosas de reflexão e de valorização das atividades e dinâmicas em desenvolvimento. Baseia-se em informações de caráter qualitativo e tem como finalidade aumentar a compreensão dos processos desenvolvidos nas dinâmicas de implementação de EpS em meio escolar, através de uma metodologia indutiva, de forma a conhecer as realidades concretas. Como os dados recolhidos são interpretados pelo investigador, admite-se que estes possam refletir a sua subjetividade (Bogdan \& Biklen, 1994). Enquanto a perspetiva quantitativa está mais relacionada com a avaliação sumativa ou de produto (resultados), a qualitativa está mais relacionada com a avaliação formativa ou de processo (Casado, 2000; Hernando, 2009).

Tendo por base o atrás referido, a avaliação qualitativa de PEpS, mais relacionada com a avaliação formativa ou de processo (Benavente, 1990; Casado, 2000; Figari, 1996; Hernando, 2009), pode ser alicerçada numa dérmache de referencialização (Figari, 1992, 1996; Figari \& Tourmen, 2006). A construção de um referencial de avaliação (isto é, o conjunto de referentes em função do quais se efetivará a avaliação) - implícito nos instrumentos de recolha de dados e no respetivo tratamento - visa a adequabilidade do processo ao contexto em que se desenrola e aos objetivos da intervenção. A referencialização de um dispositivo educativo é um conjunto de dados "complexos, diferentes e evolutivos" (Figari, 1996, p. 59) que está organizado segundo um esquema que engloba três dimensões: Induzido, Construído e Produzido (ICP). No primeiro nível, o Induzido (I), manifesta-se a função diagnóstica ou inicial, que interpreta os dados da situação; no segundo nível, o Construído (C), aclara-se a função de regulação, que determina os processos de construção - avaliação formativa ou de processo; e, por último, o Produzido $(P)$ assume a função sumativa, que acrescenta a dimensão do produzido, do impacto da ação do projeto no desenvolvimento de 
competências pessoais e sociais nos alunos e do seu reconhecimento (Capucha, 2008; Hernando, 2009).

Colocando-se em destaque o processo de desenvolvimento dos PEpS, analisa-se o nível Construído (C), que permitirá estudar, em contexto, as dinâmicas de implementação da EpS, tendo em vista caracterizar práticas de referência. Essa análise deverá considerar, nomeadamente, em que medida o programa: tornou o ambiente escolar mais seguro e saudável (dimensão ecológica); melhorou o relacionamento intra e interpessoal na escola (dimensão psicossocial); estabeleceu uma boa articulação com a comunidade extraescolar (dimensão comunitária); desenvolveu uma abordagem holística do tema, melhorou as práticas da escola, permitindo estreitar a ligação dos conteúdos programáticos à vida (dimensão curricular) (Navarro, 1999).

Para se processar a avaliação de PEpS ao nível da dimensão C (Construído), é importante produzir-se um plano estruturado (Angelmar, 1984; Capucha, 2008; Figari, 1996), identificando os atores a implicar (García, Sáez, \& Escarbajal de Haro, 2000; Sanmartí, 1990). De acordo com o referido, enumeram-se os intervenientes no PEpS, a nível externo e a nível interno - a saber: Ministério da Saúde/Ministério da Educação e Cultura (Direção Geral de Educação/Núcleo de Educação para a Saúde); Direções Regionais de Educação; Unidades de Saúde Familiar; técnicos especializados das parcerias estabelecidas; professores; pessoal não docente; alunos; pais e encarregados de educação; técnicos especializados das escolas/Agrupamentos.

A avaliação formativa ou de processo, conforme se pretende representar no esquema da Figura 1, consiste em analisar as práticas que se seguiram para implementar os princípios de uma EpS na escola determinada, tendo em consideração os objetivos propostos (Hernando, 2009). Intenta compreender os significados e o sentido do PEpS, contribuindo, assim, para o incremento da sua qualidade. Informa, ainda, dos progressos e avanços, mas também dos desajustes e vicissitudes do processo de implementação do projeto. 


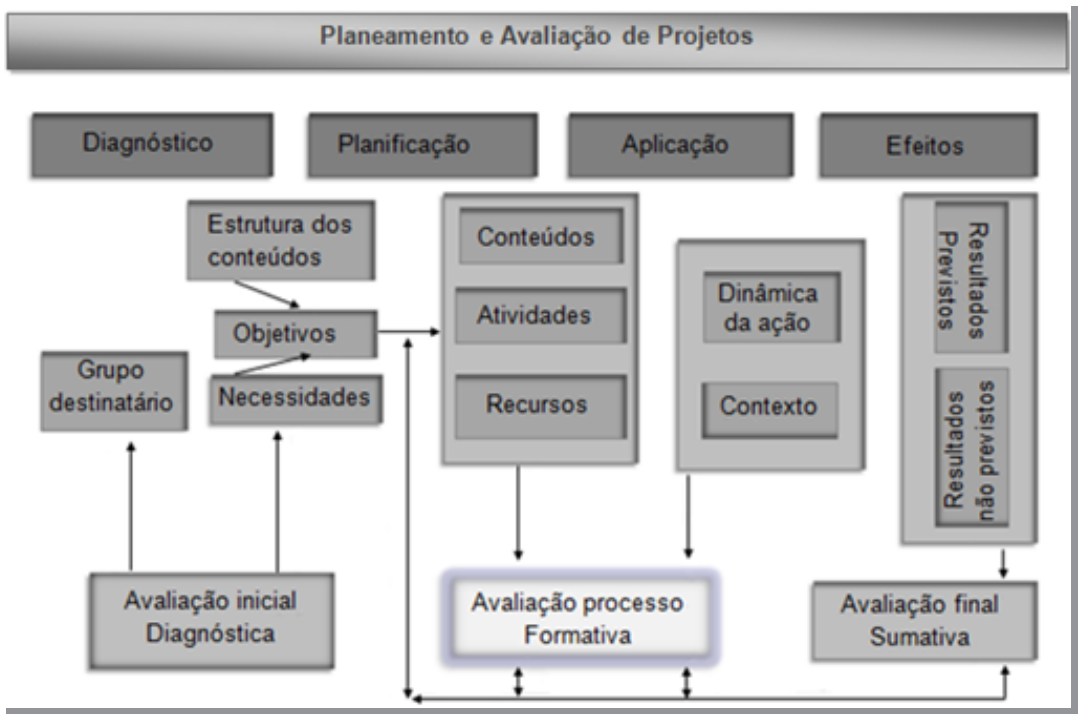

Figura 1 - Planeamento e Avaliação de Projetos (adaptado de Capucha, 2008; Figari, 1996)

\section{Metodologia}

\subsection{Opções metodológicas}

Considerando as questões de investigação formuladas, o estudo teve como objetivos: (i) desenvolver um referencial que permita avaliar práticas de referência de PEpS, no que respeita às dinâmicas de implementação; (ii) avaliar as dinâmicas de implementação de PEpS, em curso em escolas do $2^{\circ}$ e $3^{\circ} \mathrm{CEB}$, no ano de 2009/10, consideradas como práticas de referência; e (iii) fornecer elementos que, sob a forma de recomendações - proposta metodológica/guião -, estimulem a adoção de práticas de referência nos PEpS, no que concerne às dinâmicas de implementação.

Efetuou-se um estudo qualitativo construtivista, do tipo estudo de casos múltiplos de design avaliativo (Yin, 2005). Analisou-se profundamente a ação de cinco PEpS em curso em 2009/10, para os perceber em toda a sua complexidade (Stake, 2009; Yin, 2005), a partir da perspetiva dos seus atores e da interpretação do investigador, num processo dialético. O design avaliativo alicerçou-se numa démarche de referencialização, definindo-se 
critérios de qualidade múltiplos e apropriados que permitiram analisar o modo como se implementou o projeto ao nível da dimensão Construído (C) e emitir juízos de valor.

Do universo de PEpS em curso no ano letivo 2009/2010, limitou-se o estudo a uma amostra não-aleatória (Carmo \& Ferreira, 1998) de cinco escolas/Agrupamentos do $2^{\circ}$ e $3^{\circ}$ CEB, um por cada Direção Regional de Educação (DRE). Estabelecido contacto com o Núcleo de Educação para a Saúde e Ação Social Escolar - NESASE, da Direção Geral de Inovação e Desenvolvimento Curricular do Ministério da Educação, estes serviços procederam à indicação de cinco PEpS por DRE que evidenciavam práticas de referência. A seleção das cinco escolas, uma por cada DRE, teve por base serem escolas do $2^{\circ}$ e $3^{\circ}$ CEB e mostrarem disponibilidade para colaborar no estudo.

Para a recolha de dados sobre o PEpS, diversificaram-se os instrumentos de recolha de informação, de modo a que essa informação pudesse ser a mais ampla possível e obtida junto de um conjunto de atores diversificado, permitindo a triangulação dos dados, que facilitaram uma visão multifacetada, aumentando o grau de confiança nos resultados obtidos. Assim, utilizaram-se instrumentos estruturados, inquérito por entrevista, com abordagens mais informais, observação não participada e análise documental (Projeto Educativo, projeto PEpS, Plano de Atividades, plano de parcerias, relatórios/questionários elaborados pelas escolas para avaliação das atividades).

As informações obtidas foram registadas na Ficha de Registo de Dados e, após triangulação (Stake, 2009) das inferências feitas com base na análise documental e nas observações, as perceções dos intervenientes possibilitaram a sua validação. Foram objeto de um trabalho de comparação e confrontação com o Referencial de Avaliação construído, permitindo avaliar processualmente o PEpS, inscrevendo na Grelha de Avaliação a verificação ou não dos indicadores expressos.

\subsection{Démarche de referencialização}

Visando a avaliação do objeto em estudo (PEpS implementados na realidade escolar portuguesa), desenvolveu-se um referencial que permitisse avaliar práticas de referência no que respeita às dinâmicas de implementação. 
Utilizou-se, como foi já referido, a metodologia da "referencialização", que, tal como consigna Figari (2008, p. 68), se apresenta como:

procedimento que consiste em orientar, explicitar ou elaborar os referentes que presidem à descrição dos factos educativos e permitem relativizar o sentido das escolhas programáticas ou didácticas que as envolvem (concepção da acção educativa) e dos resultados que eles produzem (avaliação da acção educativa).

Na construção do modelo de referências, teve-se em conta o indicado por autores de referência, nacionais e internacionais, relativamente à implementação da EpS em meio escolar, com especial enfoque no conceito de Escola Promotora de Saúde (Loureiro, 2004; Navarro, 1995, 1999; International Union for Health Promotion and Education [IUHPE], 2009), e os estudos recentes realizados, a que se teve acesso, sobre a mesma temática (Aldinger et al., 2008; Lee et al., 2005; Mandim, 2007; Warwick et al., 2005).

O protocolo de referencialização consignou três elementos: objeto (PEpS), referencial (Referencial de Avaliação) e instrumentação (Grelha de Avaliação). Esta permitiu a verificação dos indicadores definidos, registandose a notação " $\checkmark$ " e " $x$ ", correspondente, respetivamente, ao alcance ou não dos indicadores determinados no Referencial de Avaliação, para cada uma das subdimensões em estudo.

\subsubsection{Referencial de Avaliação}

A construção do Referencial de Avaliação, entendido na aceção de Figari (1992, 1996); e (Figari \& Tourmen, 2006), compreendeu a seleção de critérios de qualidade como elementos essenciais à fundamentação dos juízos de valor manifestos e indicadores como instrumentos objetivamente observáveis e verificáveis ao serviço dos critérios.

Apresentam-se no Quadro 1 os critérios de qualidade dos PEpS, definidos com base na literatura da especialidade das áreas de confluência do estudo (projetos educacionais, promoção e educação para a saúde em meio escolar e avaliação), que se revelaram pertinentes para dar resposta às questões de avaliação formuladas e que caracterizam um 'bom' projeto. 


\section{Quadro 1 - Critérios de qualidade do PEpS}

\begin{tabular}{|ll|}
\hline Critério & Fundamentação \\
\hline Coerência & $\begin{array}{l}\text { Procura indagar o grau de adequação entre as decisões sobre o } \\
\text { funcionamento interno e o contexto externo ao projeto. }\end{array}$ \\
\hline Pertinência & $\begin{array}{l}\text { Procura verificar se os objetivos do projeto são válidos em relação aos } \\
\text { problemas/necessidades diagnosticados/as e aos interesses dos alunos. }\end{array}$ \\
\hline Eficiência & $\begin{array}{l}\text { Analisa a relação entre os resultados constatados e os meios mobilizados } \\
\text { para os atingir. }\end{array}$ \\
Efetividade & $\begin{array}{l}\text { Tenta diagnosticar os efeitos de práticas de referência nas } \\
\text { intervenções/ação do PEpS. }\end{array}$ \\
\hline Participação & $\begin{array}{l}\text { Tenta determinar o envolvimento dos intervenientes internos e da } \\
\text { comunidade educativa no projeto. }\end{array}$ \\
\hline Satisfação & $\begin{array}{l}\text { Pretende estabelecer o grau de aprovação da comunidade educativa face } \\
\text { à ação do projeto. }\end{array}$ \\
\hline
\end{tabular}

No Referencial, o objeto (PEpS), avaliado à luz do conceito de uma EpS (Loureiro, 1998, 2000, 2004; Navarro, 1995, 1999; IUHPE, 2009), é analisado nas dimensões organizacional, comunitária, ecológica, psicossocial e curricular, as quais compreendem subdimensões que funcionam como elementos a avaliar do dispositivo educativo nas cinco dimensões referenciadas, que os referentes apontam e que se privilegiou. Estas compreendem práticas de referência caracterizadas por autores de referência no processo de implementação da EpS em meio escolar, constituindo o "ideal desejável" para um PEpS. Apresenta-se, do documento global, a título de exemplo, uma das subdimensões em análise da dimensão organizacional (Quadro 2).

Cada subdimensão em análise forma um bloco coerente onde se reúnem critérios e indicadores em que se menciona um nível de aprofundamento, mais percetível, objetivo e direto, onde o indicador está, normalmente, associado ao critério existência. Completa-se com outros critérios e indicadores que dão continuidade, complementaridade, sustentabilidade e aprofundamento. Para a sua concretização, é necessária uma análise mais profunda e detalhada do PEpS em avaliação.

O documento elaborado foi enviado a três peritos da área da EpS e a um da Avaliação, para validação, que o aprovaram na generalidade, tendo sido integradas as sugestões que fizeram na especialidade. 
Quadro 2 - Estrutura do Referencial de Avaliação/Dimensão Organizacional

\begin{tabular}{|c|c|c|c|c|}
\hline \multicolumn{5}{|c|}{ Referencial de Avaliação } \\
\hline \multicolumn{4}{|c|}{ DiMENSÃo - CONSTRUÍDO (C) } & FIGARI, G. $(1996,2006)$ \\
\hline \multicolumn{5}{|c|}{ Referentes: } \\
\hline \multicolumn{5}{|c|}{ Literatura da Especialidade } \\
\hline \multicolumn{5}{|c|}{ Da Administração Central - Normativos legais } \\
\hline \multicolumn{5}{|r|}{ Do contexto local - Documentos internos } \\
\hline \multirow{14}{*}{ 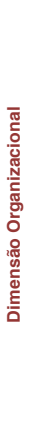 } & Sub-dimensão & Critérios & Indicadores & Autores/Normativos \\
\hline & \multirow{13}{*}{$\begin{array}{l}\text { 3. Caracterização } \\
\text { da equipa } \\
\text { educativa e } \\
\text { funções } \\
\text { desempenhadas }\end{array}$} & Existência de & O PEpS indica de forma clara e & - Colaço, C. (2008) \\
\hline & & identificação dos & estruturada todos os & - García Martínez et al. (2000) \\
\hline & & intervenientes & intervenientes na equipa & - IUHPE (2009) \\
\hline & & & & - Loureiro, I. (2000a, 2004a) \\
\hline & & & & - Rodrigues et al. (2005) \\
\hline & & & & - Sanmartí, L. (1990) \\
\hline & & Coerência no & A ação do PEpS é sujeita a & - GTES $(2005,2007)$ \\
\hline & & $\begin{array}{l}\text { acompanha-mento e } \\
\text { supervisão do projeto }\end{array}$ & $\begin{array}{l}\text { acompanhamento/orientação e } \\
\text { supervisão sistemática pelo }\end{array}$ & $\begin{array}{l}\text { - Despacho } n^{\circ} 2506 / 2007, \text { de } 23 \\
\text { de janeiro }\end{array}$ \\
\hline & & & coordenador e/ou outro. & - Lei $n^{\circ} 60 / 2009$, de 5 de agosto \\
\hline & & $\begin{array}{l}\text { Pertinência da } \\
\text { distribuição de tarefas }\end{array}$ & $\begin{array}{l}\text { A atribuição de tarefas na } \\
\text { equipa educativa é feita de }\end{array}$ & - Capucha, L. (2008) \\
\hline & & pelos intervenientes & acordo com os interesses, & - GTES $(2005,2007)$ \\
\hline & & & competências, motivação e & - Loureiro, I. (2004a) \\
\hline & & & $\begin{array}{l}\text { experiência dos recursos } \\
\text { humanos. }\end{array}$ & - Kirby et al. (2006) \\
\hline
\end{tabular}

\section{Resultados}

\subsection{Práticas de referência em PEpS verificadas no Agrupamento de escolas $A$}

Os cinco PEpS foram analisados a partir das dimensões avaliativas contempladas no Referencial de Avaliação, em resultado da triangulação da informação recolhida através da análise documental, das observações e da fundamentação da análise das entrevistas, registando-se, na Grelha de Avaliação, a verificação ou não do indicador com a notação " $\checkmark$ " e " $x$ ", respetivamente.

O estudo evidenciou práticas consideradas de referência nas dinâmicas de implementação dos cinco PEpS. Contudo, só o PEpS do Agrupamento de Escolas A, pertencente à DREN, registou os critérios de qualidade que se definiram. Apresenta-se, no Quadro 3, a título de exemplo, parte da sua Grelha de Avaliação, efetuada para a subdimensão "Caracterização da equipa educativa e funções desempenhadas" da dimensão organizacional. 


\section{Quadro 3 - Grelha de Avaliação do PEpS do Agrupamento de Escolas A na subdimensão "Caracterização da equipa educativa e funções desempenhadas"/Dimensão Organizacional}

\begin{tabular}{|c|c|c|c|c|c|c|}
\hline & \multirow[b]{2}{*}{ Sub-dimensão } & \multirow[b]{2}{*}{ Critérios } & \multirow[b]{2}{*}{ Indicadores } & \multicolumn{2}{|c|}{ Avaliação } & \multirow[b]{2}{*}{ Observações } \\
\hline & & & & $\begin{array}{c}\text { Análise } \\
\text { documental }\end{array}$ & Entrevista & \\
\hline$\frac{\overline{\mathrm{d}}}{\mathrm{C}}$ & \multirow{3}{*}{$\begin{array}{l}\text { 3. Caracterização } \\
\text { da equipa } \\
\text { educativa e } \\
\text { funções } \\
\text { desempenhadas }\end{array}$} & $\begin{array}{l}\text { Existência de } \\
\text { identificação dos } \\
\text { principais } \\
\text { intervenientes }\end{array}$ & $\begin{array}{l}\text { O PEpS indica de forma } \\
\text { clara e estruturada todos } \\
\text { os intervenientes na } \\
\text { equipa educativa. }\end{array}$ & & $\mathbf{P}$ & $\begin{array}{l}\text { Sem registo } \\
\text { escrito }\end{array}$ \\
\hline 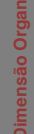 & & $\begin{array}{l}\text { Coerência no } \\
\text { acompanha- } \\
\text { mento e } \\
\text { supervisão do } \\
\text { projeto }\end{array}$ & $\begin{array}{l}\text { A ação do PEpS é sujeita } \\
\text { a } \\
\text { acompanhamento/orientaç } \\
\text { ão e supervisão } \\
\text { sistemática pelo } \\
\text { coordenador e/ou outro. }\end{array}$ & $\mathbf{P}$ & $\mathbf{P}$ & \\
\hline & & $\begin{array}{l}\text { Pertinência da } \\
\text { distribuição de } \\
\text { tarefas pelos } \\
\text { intervenientes }\end{array}$ & $\begin{array}{l}\text { A atribuição de tarefas na } \\
\text { equipa educativa é feita de } \\
\text { acordo com os interesses, } \\
\text { competências, motivação } \\
\text { e experiência dos recursos } \\
\text { humanos. }\end{array}$ & & $\mathbf{P}$ & $\begin{array}{c}\text { Sem registo } \\
\text { escrito }\end{array}$ \\
\hline
\end{tabular}

A avaliação global permitiu evidenciar práticas de referência caracterizadas no Referencial de Avaliação nas dinâmicas de implementação do referido $P E p S$, que interessa realçar:

- integração da EpS no Projeto Educativo, visando a concretização de uma EpS;

- elaboração de um diagnóstico/inventariação de necessidades, seleção de prioridades;

- elaboração/adequação de um plano de ação relevante face às necessidades diagnosticadas;

- equipa educativa multidisciplinar coesa/forte liderança; identificação dos intervenientes no projeto;

- desenvolvimento do trabalho de equipa e clarificação dos níveis de motivação, interesse e responsabilidade;

- intervenções para grupos-alvo em detrimento de intervenções individuais;

- práticas que visam a promoção da educação alimentar (apoio no refeitório, merenda escolar); 
- intervenções que visam a capacitação/empowerment dos alunos, professores, funcionários e pais;

- utilização de uma visão holística da saúde na metodologia de abordagem da EpS nas atividades desencadeadas;

- trabalho colaborativo com pais, alunos e profissionais de saúde;

- colaboração dos alunos líderes (educação por pares), na educação alimentar, na promoção da atividade física, na gestão de emoções e na dinamização do Gabinete do Aluno;

- dinamização ativa do Gabinete de Apoio ao Aluno, criando estruturas de acompanhamento/aconselhamento dos alunos;

- estabelecimento de parcerias, principalmente com os serviços de saúde locais (saúde pública, centro de saúde - Unidade de Saúde Familiar, hospital e farmácia), otimizando recursos para a realização de colóquios, workshops, e apoio no Gabinete do Aluno, aumentando a cooperação e partilha de experiências;

- plano de avaliação global do projeto centrado no processo, possibilitando a (re)estruturação do projeto - apresentação de relatório descritivo.

\subsection{Síntese: Liderança e coesão}

Face aos resultados encontrados, considera-se que a qualidade evidenciada no PEpS do Agrupamento de Escolas A configura uma forte liderança e coesão que se manifesta no alcance dos critérios definidos para um 'bom' projeto, nomeadamente: coerência, pertinência, eficiência, efetividade, participação e satisfação.

\section{Considerações finais}

As intervenções em meio escolar que visem a capacitação (empowerment) dos alunos contribuem para a sua formação integral, potenciando a adoção de estilos de vida saudáveis, um efetivo sucesso escolar e o desenvolvimento de competências para o exercício da cidadania (Ministério da Educação/GTES, 2007; IUHPE, 2009; Matos, 2005). Reconhecendo o papel determinante da escola nesta área, importa que se 
implemente em todos os Agrupamentos de Escolas um projeto global que deverá assentar nos princípios do movimento de EpS. Consagra-se, assim, que deverá continuar a ser feita uma forte aposta na implementação da EpS no contexto escolar português.

Tal como refere Hernando (2009), crê-se que o modo como se implementou/organizou o PEpS será determinante para a efetividade das intervenções e para o impacto positivo que este tem junto das crianças e adolescentes na promoção da saúde e de estilos de vida saudáveis. A avaliação interna desses projetos é um ato complexo de realizar, dado que implica um trabalho delicado e subtil, intimamente articulado com o ritmo de evolução das atividades, capaz simultaneamente de uma proximidade do terreno e de um distanciamento crítico. Pugna-se pela sua efetividade, a qual deve ser complementada, sempre que possível, com ações avaliativas externas. Neste sentido, a avaliação externa de cinco PEpS preconizada, alicerçada no Referencial de Avaliação que se construiu, o qual se apresenta como um referencial possível na avaliação dos referidos projetos, assume-se em complementaridade da avaliação interna, dando coerência ao processo de implementação da EpS no meio escolar.

Registe-se, ainda, que o modelo de referência construído permitiu definir a imagem antecipatória de um processo de transformação do real, no sentido de Barbier (1996), relativamente à démarche de elaboração de um projeto, o que contribuiu para a concretização do "ideal desejável" de um PEpS, passos que se consagram no sentido da inovação nesta área.

Contribuiu-se, no estudo realizado, para a pesquisa de práticas de referência já caracterizadas e/ou outras que permitiram construir o documento "Práticas de referência em PEpS - Guião de implementação em meio escolar". Este será divulgado oportunamente, constituindo-se como um contributo que pode potenciar a articulação entre teoria e prática no que respeita às dinâmicas de implementação e/ou desenvolvimento dos PEpS das escolas/Agrupamentos portugueses, possibilitando (re)definir o processo de implementação da EpS, logo adequar os projetos aos contextos específicos, tornando-os relevantes para cada realidade.

O estudo realizado, além de contribuir para a avaliação da qualidade dos PEpS implementados na realidade escolar portuguesa e explicitar as suas dinâmicas, concorre, também, para a necessária reflexão mais alargada 
que se impõe, nesta área, visando auxiliar os decisores políticos na implementação de medidas que visem a efetiva integração da EpS em contexto educativo.

\section{Referências}

Aldinger, C., Xin-Wei, Z., Li-Qun, L., Jun-Xiang, G., Sen Hai, Y., \& Jones, J. (2008). Strategies for implementing health-promoting schools in a province in China. Promotion \& Education, 15(1), 24-29. Retirado de http://ped.sagepub.com/ cgi/reprint/15/1/24

Angelmar, R. (1984). Les méthodes de l'évaluation. In J. P. Nioche \& R. Poinsard (Eds.), L'évaluation des politiques publiques. Paris: Economica.

Barbier, J. M. (1996). Elaboração de projectos de acção e de planificação. Porto: Porto Editora.

Benavente, A. (1990). Avaliação e inovação educacional. Inovação, 3(4), 33-46.

Bogdan, R., \& Biklen, S. (1994). Investigação qualitativa em educação. Porto: Porto Editora.

Capucha, L. (2008). Planeamento e avaliação de projectos - Guião prático. Lisboa: DGIDC.

Cardinet, J. (1976). L'élargissement de l'évaluation. Neuchâtel: IRDP.

Carmo, H., \& Ferreira, M. (1998). Metodologia da investigação - Guia para autoaprendizagem. Lisboa: Universidade Aberta.

Casado, R. P. (2000). Acción de evaluar. In A. F. Osuna (Coord.), Salud pública y Educación para la Salud. Madrid: Elsiever.

Costa, J. A. (2007). Projectos em educação: Contributos de análise organizacional. Aveiro: Universidade de Aveiro.

Figari, G. (1992). Para uma referencialização das práticas de avaliação. In A. Estrela \& A. Nóvoa (Org.), Avaliações em educação: Novas perspectivas. EDUCA. Lisboa.

Figari, G. (1996). Avaliar: Que referencial? Porto: Porto Editora.

Figari, G. (2008). A avaliação da escola: Questões, tendências e modelos. In M. P. Alves \& E. A. Machado (Org.), Avaliação com sentido(s): Contributos e questionamentos. Santo Tirso: De Facto Editores.

Figari, G., \& Tourmen, C. (2006). La référentialisation: Une façon de modéliser l'évaluation de programme, entre théorie et pratique. Vers une comparaison des approches au Québec et en France. Mesure et évaluation en éducation, 29(3), 5-25.

García, A, Sáez, J., \& Escarbajal de Haro, A. (2000). Educación para la Salud: La apuesta por la calidad de vida. Madrid: Arán Ediciones S. A.

Hadji, C. (1994). Avaliação, regras do jogo. Das intenções aos instrumentos. Porto: Porto Editora. 
Hernando, M. A. (2009). Los proyectos y los programas en EpS. In P. R. Quesada (Coord.), Promoción y Educación para la Salud - Tendencias innovadoras. Madrid: Ediciones Díaz de Santos.

International Union for Health Promotion and Education (IUHPE) (2009). Construindo escolas promotoras de saúde: Directrizes para promover a saúde em meio escolar. Retirado de http://www.iuhpe.org/uploaded/Publications/ Books_ Reports/HPS_Guidelinesll_2009_Portuguese.pdf

Kahan, B., \& Goodstadt, M. (1998). An exploration of best practices in health promotion: A short history of the Best Practices Work Group - Centre for Health Promotion, University of Toronto. Retirado de http://www.idmbestpractices.ca

Lee, A., Leger, L., \& Moon, A. (2005). Evaluating health promotion in schools: A case study of design, implementation and results from the Hong Kong Healthy Schools Award Scheme. Promotion \& Education. Retirado de http://ped.sagepub.com/cgi

Loureiro, I. (2004). A importância da educação alimentar: O papel das escolas promotoras de saúde. Revista Portuguesa de Saúde Pública, 22(2), 43-55.

Loureiro, I. (Coord.). (1998). A rede nacional das escolas promotoras de saúde. Lisboa: CCPES - Comissão de Coordenação da Promoção e Educação para a Saúde, Ministério da Educação.

Loureiro, I. (Coord.). (2000). O que é a saúde na escola. Guião orientador. Escolas promotoras de saúde ( $1^{a}$ ed.). Lisboa: CCPES - Comissão de Coordenação da Promoção e Educação para a Saúde, Ministério da Educação.

Mandim, J. F. O. (2007). As dinâmicas para a promoção da saúde numa escola do Ensino Básico do Porto: Um estudo de caso (Tese de Mestrado). Universidade Aberta, Porto.

Matos, M. G. (Ed.). (2005). Comunicação, gestão de conflitos e saúde na escola. Lisboa: Edições FMH.

Mendonça, M. (2002). Ensinar e aprender por projectos. Cadernos CRIAP, n 31 . Porto: Edições Asa.

Ministério da Educação - Grupo de Trabalho de Educação Sexual (GTES) (2007). Educação para a Saúde - Relatório final. Retirado de www.dgidc.min-edu.pt

Navarro, F. (1995). Modelos de intervenção em saúde na educação pré-escolar e nos ensinos básicos e secundário. In A Educação para a Saúde. Papel da Educação Física na promoção de estilos de vida saudáveis: Simpósio. Lisboa: Programa de Promoção e Educação para a Saúde.

Navarro, F. (1999). Educar para a saúde ou para a vida? Conceitos e fundamentos para novas práticas. In AAVV. Actas do I Congresso Nacional de Educação para a Saúde (pp. 13-28). Braga: Universidade do Minho.

Nóvoa, A., Almeida, C., \& Le Boterf, G. (1993). A avaliação participativa no decurso dos projectos: Reflexões a partir da prática de uma experiência de terreno. In A. Estrela \& A. Nóvoa (Org.), Avaliação em educação: Novas perspectivas (pp. 105-123). Lisboa: INIC. 
Rodrigues, P. (1995). As três "lógicas" da avaliação de dispositivos educativos. In A. Estrela \& P. Rodrigues (Orgs.), Para uma fundamentação da avaliação em educação. Lisboa: Edições Colibri.

Sanmartí, L. S. (1990). Educación Sanitária: Principios, métodos, aplicaciones. Madrid: Ediciones Díaz de Santos.

Stake, R. (2009). A arte da investigação com estudos de caso. Lisboa. Fundação Calouste Glubenkian.

Warwick, I., Aggleton, P., Chase, E., Schagen, S., Blenkinsop, S., Schagen, I., Scott, E., \& Eggers, M. (2005). Evaluating healthy schools: Perceptions of impact among school-based respondents. Health Education Research: Theory \& Practice, 20(6), 697-708. Retirado de http://her.oxfordjournals.org/cgi/reprint/20/6/697

Yin, R. K. (2005). Estudo de caso. Planejamento e métodos ( $3^{\mathrm{a}}$ ed.). Porto Alegre: Artmed. 


\title{
HEALTH EDUCATION PROJECTS IN SCHOOLS: FROM EVALUATION TO REFERENCE PRACTICES
}

\begin{abstract}
Planning pertinent and efficient interventions within Health Education (HE) in schools determines the implementation of evaluation procedures that should not only translate an analysis of the developed action, but also contribute for a reflection about the implementation process of HE in Portuguese schools. This, in fact, aims for a search of a qualitative optimization of the interventions. According to the previous assumptions, we intend to establish a link between the theoretical analysis and the practical reflection, on the basis of a concrete practice. Therefore, the present article has the following main objectives: (i) to present the evaluation model used in a multicases study of qualitative nature: "School, Health and Society: Evaluation Studies of Health Education Projects", which was developed between 2009/2011; and (ii) to disseminate the reference practices related to the dynamics of one Health Education project, which evidenced to correspond to the defined quality criteria for a 'good' project.
\end{abstract}

Keywords

School; Health Education projects; Evaluation; Reference practices

\section{PROJETS D'ÉDUCATION POUR LA SANTÉ DANS LES ÉCOLES: DE L'ÉVALUATION AUX PRATIQUES DE RÉFÉRENCE}

\section{Résumé}

La planification d'interventions pertinentes et efficaces, au sein de l'Éducation pour la Santé (EPS) dans les écoles, détermine la mise en œuvre des procédures d'évaluation qui se traduisent, pas seulement par un solde d'action élaboré, mais aussi par la contribution pour réfléchir sur le processus de mise 
en œuvre de I'EPS dans l'école portugaise à la poursuite d'une optimisation qualitative des interventions. À la lumière de cette présupposition, et en établissant un lien entre l'analyse théorique et la réflexion à partir d'une pratique concrète, cet article vise: (i) présenter le modèle d'évaluation établi dans l'étude de cas pluriels de nature qualitative "École, Santé et Société: Études d' Évaluation des Projets d'Éducation pour la Santé", que l'on a développé entre 2009/2011; (ii) diffuser des pratiques de référence sur la dynamique de l'un des projets de l'Éducation à la Santé impliqués dans l'étude, et qui a montré des critères de qualité définis pour la construction d'un 'bon' projet.

Mots-clé

École; Projets d'Éducation à la Santé; Évaluation; Pratiques de référence

Recebido em Dezembro/2011 Aceite para publicação em Maio/2013 